\title{
OperaBLE: An IoT-Based Wearable to Improve Efficiency and Smart Worker Care Services in Industry 4.0
}

\author{
Luis Roda-Sanchez $\mathbb{D}^{1},{ }^{1}$ Celia Garrido-Hidalgo $\left(\mathbb{D},{ }^{1}\right.$ Diego Hortelano $\mathbb{D},{ }^{1}$ Teresa Olivares $\left(\mathbb{D},{ }^{2}\right.$ \\ and M. Carmen Ruiz $\mathbb{1}^{2}$ \\ ${ }^{1}$ Albacete Research Institute of Informatics (I3A), Albacete 02071, Spain \\ ${ }^{2}$ University of Castilla-La Mancha (UCLM), Albacete 02071, Spain \\ Correspondence should be addressed to Diego Hortelano; diego.hortelano@uclm.es
}

Received 23 February 2018; Revised 17 June 2018; Accepted 9 July 2018; Published 26 August 2018

Academic Editor: Mucheol Kim

Copyright ( 2018 Luis Roda-Sanchez et al. This is an open access article distributed under the Creative Commons Attribution License, which permits unrestricted use, distribution, and reproduction in any medium, provided the original work is properly cited.

\begin{abstract}
Industry 4.0 is leading the Fourth Industrial Revolution transforming traditional factories into smart factories governed by the Internet of Things (IoT). In order to assist smart factory employees, this paper introduces OperaBLE, a Bluetooth Low Energy (BLE) wearable proposal which is aimed at enhancing working conditions and efficiency in Industry 4.0 scenarios. We have developed two innovative algorithms for OperaBLE focused on power awareness as the key-enabling attribute towards success: Low-Frequency Movement Characterisation Algorithm (LoMoCA) and Adaptive Heart Rate Algorithm (AHRA). Novel experiments have been carried out using OperaBLE to determine its operability, reliability, and lifespan. Results obtained during experimentation demonstrate how OperaBLE empowers human-machine collaboration embedding workers in closed-loop performance and ensuring nonharmful working conditions by means of power-aware algorithms. OperaBLE is due to bring digitalisation into smart factories, playing an essential role in the emerging wearable revolution to arise in the following years towards smart production systems.
\end{abstract}

\section{Introduction}

The Fourth Industrial Revolution has arisen to transform the current industry model and to introduce digitalisation into traditional factories improving production rates and promoting collaboration. This emerging revolution is the widely known Industry 4.0 [1]. The 4.0 attribute focuses on the Internet of Things (IoT) [2] applied to industrial systems in order to interconnect objects, machines, and humans in smart factories. Collaborative tasks are encouraged by means of increasing production rates and minimising costs. This is achieved by introducing cyberphysical systems (CPS) [3], perceived as the embeddedness of sensors to collect data and perform cognitive algorithms through the Internet.

Sustainability is one of the greatest concerns for this emergent paradigm. In a few years, factories will be immersed into IoT ecosystems composed of heterogeneous networks, which is the reason why power-aware alternatives are mandatory to avoid an additional energy waste. Not only environmental but also social sustainability is encouraged to face the greatest challenges of Industry 4.0. New CPS are due to be transformed into human-centric architectures focused on employees which ensure security, wellness, and comfort towards optimal working conditions and production rates.

The recent proliferation of the wearable market is a key indicator of global success based on the embeddedness of sensors and actuators to enable the development of smarter devices. On one hand, technologies such as Bluetooth Low Energy (BLE) have taken the lead in terms of IoT standardization due to the necessity of greener and more flexible alternatives. Particularly, BLE is leading an emergent industrial transformation due to the recent adoption of BLE-mesh topology, where broadcast capability will play an important role. On the other hand, accelerometers are setting the beginning of a new IoT revolution enabling gesture-based systems to enable a better interaction between humans and machines. 
These devices generate huge amounts of data which are due to be processed and filtered to extract specific information, which is a global challenge widely known as big data.

OperaBLE is introduced in this paper in order to face the major challenges of Industry 4.0. This BLE-based prototype of work band is intended to bring digitalisation into smart factories and to enhance working conditions by immersing employees into CPS, taking into account human care services for workers.

It consists of a modular wearable that improves security in industrial environments, being able to predict harmful situations or even working accidents. This low-power wearable will impact positively on the industrial paradigm due to its wide range of novel applications for the Industrial Internet of Things (IIoT). Being focused on movement characterisation techniques and heart rate measurements, OperaBLE uses learning skills to track operators in order to improve the industry value chain from a power-aware perspective.

The novelty of this paper lies on the introduction of adaptive algorithms specifically developed for OperaBLE, which play an essential role in its applicability to the incoming industrial paradigm. In addition, the experiments carried out during this investigation permitted a technical evaluation of OperaBLE according to user performance, success rates, and energy consumption with promising results. The rest of this paper is organized as follows: Section 2 highlights related works. Section 3 describes OperaBLE, focusing on hardware in Section 3.1 and detailing the algorithms developed in Sections 3.2 (LoMoCA) and 3.3 (AHRA). Section 4 introduces the experiments carried out and discusses the results. Eventually, Section 5 offers the remarkable conclusions of this research and proposes future works.

\section{Related Works}

Accelerometer-based wearables are specially used to keep a track of information about personal activities and their energy consumption. The number of commercial wearables (usually wristbands identified as smart bands) has increased in last years. Regarding academic and research works, they can be classified in different topics: medical and rehabilitation, movement recognition, older adults, sports, positioning, and work.

Concerning medical aspects, a complete system to avoid the hospital acquired infection is proposed in [4], using sensors and wristbands equipped with accelerometers. In a similar way, other smart bands are proposed for muscular movement recognition and physiological signals [5], for Parkinson's disease detection [6] and for helping patients with obstructive sleep apnea [7]. Additionally, accelerometer-based devices are widely used for rehabilitation applications [8].

Gesture and movement recognition is a wide research field concerning devices based on accelerometers. This recognition can be performed by wearables [9] or even by smartphones [10]. It uses accelerometers to assist older adults; in [11], authors present an accelerometer for movement monitoring; in [12], a method based on accelerometers for identification of declining balance is proposed; finally,
TABle 1: Principal attributes of OperaBLE.

Attributes
Zero fails
Sustainability
Cooperation
Standardization
Human in the loop
Health
Intelligence

accelerometers are used to detect some predefined movements (stand-to-sit and sit-to-stand) in young and old adults [13]. Furthermore, in [14], the authors design and implement an embedded system to estimate the pedestrian walking position.

Finally, regarding the use of accelerometers at workplaces, most of articles are focused on the construction sector to enhance security at work. In [15], commercial accelerometers are used to measure the trunk flexion and lateral bending angle in laboratory conditions; this experiment is repeated in a real construction environment in [16]. Both studies are focused on the analysis of the data collected using commercial accelerometers. In [17], an approach based on supervised motion tensor decomposing for construction workers is presented. In [18], authors use the integrated sensor of mobile phones for activity recognition of construction workers. However, other studies are focused on different workers; for example, in [19], authors explore the utility of accelerometers for determining sitting time in office workers. Despite the fact that the use of accelerometers has been evaluated for different professionals, it has not been taken into account for industrial environments to the date, where it could highly contribute to the new Industry 4.0 .

\section{OperaBLE: The Proposed Wearable}

This investigation introduces a wearable intended to satisfy IoT requirements in smart factories. The main attributes of the wearable prototype, OperaBLE, are listed in Table 1. It is an autonomous device able to learn behavioural patterns from individuals and, thus, enhance their working conditions. Although a specific hardware is proposed in this work to implement an evaluable wearable, the major 


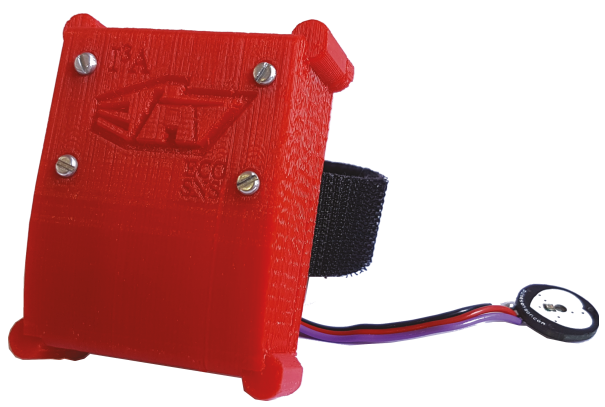

(a) Exterior design: OperaBLE with pulse sensor

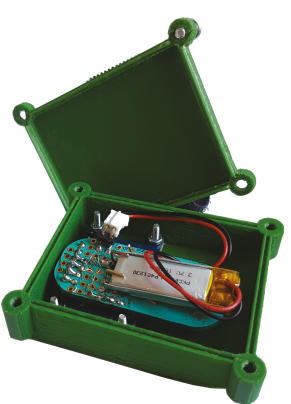

(b) Interior design: OperaBLE with accelerometer

Figure 1: OperaBLE, prototype used for experimentation.

contributions of OperaBLE lie in its functionality and, thus, in the proposed algorithms.

For the development of OperaBLE, different hierarchies have been established, being the first one its reliability for Industry 4.0 applications and the second its sustainable operation. Thus, a right functionality is prioritized over energy efficiency in case of conflict. The key challenge behind OperaBLE is to enable the implementation of its functionality with a high degree of reliability in any lightweight IoT device. This promises to save as much energy as possible due to the reduced microcontroller operating frequency that characterises most tiny IoT devices, which is complimented with the appropriate management of sensors and communication modules.

A technical description of the hardware used to implement the functionality of OperaBLE is provided in Section 3.1. The development of a low-frequency movement characterisation algorithm called LoMoCA, a remarkable contribution of this paper, is addressed in Section 3.2. Moreover, a sustainable algorithm able to adapt the heart rate sampling frequency to each worker, AHRA, is introduced in Section 3.3. Again, the major achievements of this paper are based on the power-aware approach used to develop the mentioned algorithms, which enable the functionality proposed to be run by in lightweight IoT devices typically operating with reduced microcontroller frequencies.

3.1. Hardware Components. This section describes the hardware used to develop the OperaBLE prototype. The core system is the controller board which has an integrated $B L E$ radio module used to transmit data. The device used is LightBlue Bean [20], a low-frequency board that includes I2C and SPI interfaces combined with multiple I/O pins. Regarding integrated sensors, it integrates a temperature sensor and a three-axis accelerometer. Finally, a low-frequency microcontroller, working at $8 \mathrm{MHz}$, is responsible for managing all parts.

Despite having a three-axis accelerometer integrated in the microcontroller platform, we have assembled an inertial measurement unit (IMU). We included this new board because it has ten degrees of freedom: three-axis accelerometer, three-axis gyroscopes, three-axis magnetometer, and a pressure sensor. Although in this paper we use only the accelerometer and the gyroscope, it enables the use of extra sensors that can be used for further investigation. In addition, a pulse sensor has been included in our prototype to measure the heart rate of operators and to identify harmful situations for them. Eventually, as envelope for the prototype, we have designed a housing using 3D-printing techniques, modelled with the shape shown in Figures 1(a) and 1(b).

One of the main attributes of OperaBLE prototype is its modularity, which is the reason why functionalities to be developed should be tested under different conditions. Our case studio was carried out using two different OperaBLE designs (see Figure 1) for testing individually the two algorithms developed. Considering these characteristics, OperaBLE is a combination of low-frequency and low-cost prototyping board for developing algorithms based on adaptability, low power operation, and sampling frequency.

3.2. Movement Characterisation. This section describes in detail the algorithmic development that permits OperaBLEcharacterised movements. In the first place, it is necessary to underline that movement recognition based on accelerometers is not a trivial task, since they provide raw acceleration data that has to be properly filtered and interpreted. For instance, a high difficulty is noticed while separating accurately the body movements and gestures from gravity, for which data sensed by accelerometers is expected to be processed by demanding algorithms that require exhaustive processing and analytics.

As mentioned previously, one of the key achievements of this investigation is a system development for movement recognition using a sampling frequency around $10 \mathrm{~Hz}$, which is up to ten times lower than the one used in novel studies. This fact provides great advantages towards a more efficient battery usage, being highlighted in the following lines the most remarkable contributions:

(1) The movement characterisation algorithm can be implemented in a wide range of devices, including those governed by low-frequency microcontrollers which are less energy-demanding than highfrequency computational platforms [21]. Thus, the minimisation of resources required by this algorithm to process raw acceleration data and identify specific body movements enables its implementation in lightweight and cost-effective devices, contributing towards a more sustainable use of resources. 
(2) The algorithm itself, apart from being able to achieve a successful characterisation with a low number of samples per movement, requires as a result a low measurement duty cycle. Downsampling is not an essential energy-saving source of OperaBLE but contributes to the minimisation of energy consumed by accelerometers through the reduction of data acquired.

(3) The use of BLE technology to perform communication enables the optimisation of energy consumed by the communication module as well. A fundamental idea behind the movement characterisation algorithm is that, while data being acquired is not of interest for OperaBLE (which preprocesses raw acceleration data), no information is transmitted to the processing stage. What BLE enables is the possibility to perform sleeping periods between data transmissions with negligible consumption and, as a result of this preprocessing proposed, the amount of data being encapsulated and sent via BLE is greatly reduced.

Although the reduction of either sensor duty cycle or BLE transmissions enhances the energy efficiency of the device, again, the most remarkable contribution to battery saving is provided by the reduced microcontroller frequency demanded by the algorithm. To satisfy the reliability challenge with this low-frequency restriction, we propose to increase the number of different data sources to be analysed. Considering that accelerometers provide data in each space direction, the addition of gyroscopes provides a total of 6 degrees of freedom (DOF). Therefore, we obtain more data to enhance the reliability of the system while low power consumption can be noticed thanks to a low-frequency microcontroller operation required by devices where this algorithm is expected to be implemented.

3.2.1. LoMoCA: Low-Frequency Movement Characterisation Algorithm. This section expands the procedures performed by our algorithm, LoMoCA (see the flow diagram shown in Figure 2 including representative stages). The algorithm of OperaBLE is divided into two parts, differentiated as implementation in a wearable device (preprocessing) and in a nonwearable device (processing).

Regarding the wearable device, we have developed an optimised code to save battery that maintains the device in sleep mode while no significant movement is noticed as can be seen in Figure 2(1). By the time OperaBLE identifies the beginning of a new movement, the subroutine acquires data from the accelerometer and gyroscope integrated in the IMU and stores them in the device until the end of the movement. This movement start event is triggered by a threshold, comparing constantly the current and the previous sample to verify if a difference greater than $1 \mathrm{~m} / \mathrm{s}^{2}$ (acceleration) or $1 \mathrm{rad} / \mathrm{s}$ (angular velocity) takes place. To determine the end of each movement, the device enters on standby mode while waiting for a significant acceleration or angular velocity during a second. If no movement is perceived during this second, the data collection procedure is considered to be finished.
As stated previously, the acquisition of movement data continues until the previous condition stops being satisfied. Then, the device has two data sources which are checked: accelerometer data (3 DOF) or accelerometer and gyroscope data $(6 \mathrm{DOF})$. The use of $6 \mathrm{DOF}$ considers both angular velocity and acceleration data, which are encoded and sent to the nonwearable device when relevant, see Figure 2(2).

The processing in nonwearable device begins with the data reception event. By the time the analysis stage in Figure 2(3) starts, OperaBLE checks the mode of operation selected in advance. After that, data are decoded properly to proceed to the movement recognition stage. LoMoCA uses a method to recognise movements based on patterns. Thanks to its flexibility, it allows the introduction of new patterns depending on the kind of industry or task that the operator performs. In the first stage, the program checks all patterns stored in a nonwearable device to take into account all of them and compare them with the movement. If the user needed to store a new movement, OperaBLE would be prepared to store it without requiring a reset since the movement recognition procedure also considers patterns recently stored.

At this point, the next analysis procedure is conducted once for each axis and, thus, for each pattern. The most crucial stage is the so-called normalisation of movements (identified in Figure 2(4)). For this, it is necessary to refer the dynamic time warping (DTW) algorithm, since LoMoCA is based on it to evaluate similarity between each pattern and the movement executed. This recursive algorithm was introduced in [22] with the aim of improving existing speech recognition procedures. It is widely used nowadays and is based on the principle of comparing two time series instead of comparing two data sets according to their Euclidean distance (sample by sample). DTW calculates the so-called distance matrix [23], which considers either the comparison of a pair of samples belonging to a time series as well as the adjacent values in order to reach the smallest path and, consequently, the most effective alignment of two time series. The library used during the experiments for applying the DTW algorithm can be accessed in [24]. However, since we noticed that the accuracy of DTW was not enough to achieve a proper movement characterisation with lowfrequency sampling, we developed a proprietary algorithm based on DTW, where several issues noticed during the investigation were solved.

In order to carry out the normalisation procedure, axes of patterns are classified in advance. This classification determines which axes are relevant or not in the movement, and then, normalisation plays an important role since DTW is not useful to compare sequences with different amplitudes. Regarding normalisation, for each axis, if the amplitude of acceleration or angular velocity is higher than $4 \mathrm{~m} / \mathrm{s}^{2}$ or $4 \mathrm{rad} / \mathrm{s}$, respectively, this axis is normalised using the maximum absolute value in the entire sample. Once this procedure is performed for every axis received, the algorithm compares the concordance between pattern and the normalised movement. Firstly, if the normalisation of the pattern and the movement does not match, the algorithm determines that the incoming movement does not coincide with that pattern. In 


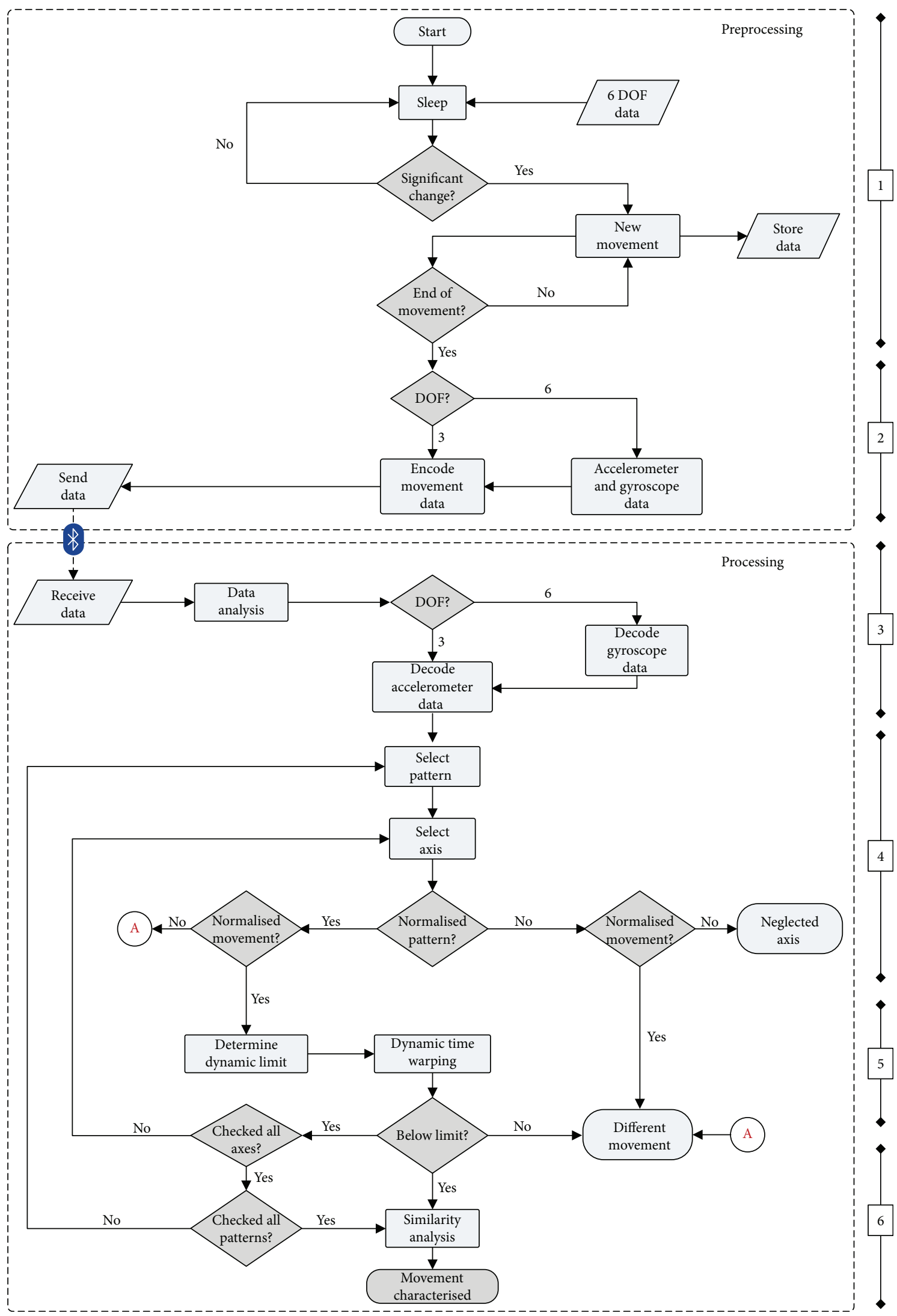

Figure 2: Processing stages of LoMoCA. 
the case where pattern and movement are not normalised, the algorithm considers that this axis is not relevant in the movement and discards it for the final decision. Conversely, when the same axes in the pattern and in the sample result to be normalised, the sample is assumed to be a clear candidate to match with the pattern stored. When all axes are analysed in terms of normalisation, the characterisation can continue to the next stage, the dynamic limit determination.

In the next stage (in Figure 2(5)), the dynamic limit is determined according to different parameters such as the length of movement and pattern. For this, an experimental study was conducted to select a dynamic limit which represented similarity between patterns and samples. Equation (1) shows the experimental setting of the parameters used to check similarity, where the threshold is adjusted in each situation depending on the number of samples per movement and pattern considered $(N)$. As can be observed in (1), the limit reaches a constant value for data sets composed of more than 15 samples. Then, DTW is applied to normalised movements and obtain a value of similarity. If all relevant axes in each movement-pattern pair are below the dynamic limit established, a potential compatibility has been found.

$$
\text { Limit }_{\text {dynamic }}=38 \cdot N+30 \text {. }
$$

Eventually, when all patterns have been checked (in Figure 2(6)), a global similarity analysis is performed to select (from all movement patterns) the closest match and the movement is characterised. For this, the sum of all differential factors obtained (one per each axis analysed in an entire pattern) is taken into account and it is compared with the resulting sums of the other patterns. To finish characterisation, LoMoCA selects the smallest sum as the better pattern-movement match, since the differential factor decreases when similarity increases.

This work provides experiments to discuss the advantages and disadvantages of using accelerometers combined with gyroscopes over using only accelerometers, since a higher energy is typically demanded by gyroscopes. However, the major focus of this section lies in enabling the implementation of the movement characterisation algorithm in lightweight devices with low-frequency microcontrollers, contributing to power-aware approaches.

LoMoCA is a complex algorithm because of the large number of variables to be managed. However, since it is implemented using several filters accurately adjusted, it does not require high computational power. It is important to highlight that LoMoCA is designed for characterising long and short movements, being the last ones is a challenge for most existing movement characterisation algorithms due to the reduced number of samples. OperaBLE is transparent to end users and, hence, ideal to overcome the existing technological gap between digitalised and nondigitalised users. Following this idea, the system implemented is able to learn new movements with no need to modify the software or make changes in the characterisation algorithm, which is an important attribute of OperaBLE.
3.3. Well-Being and Security of Workers. Human-centred architectures are being promoted by means of focusing on the social dimension of sustainability. Apart from the ability for movement characterisation using our low-frequency algorithm, OperaBLE is responsible for assuring nonharmful working environments for factory workers, guaranteeing the prevention of human damages.

OperaBLE has been developed to be embedded in body area networks (BAN) composed of cognitive clothes. Since the focus of this paper is the OperaBLE wearable, the complete BAN is framed by previous studies [25].

In terms of human well-being, OperaBLE measures heart rate in order to monitor unexpected changes with respect to a reference value established during calibration for each worker. We introduce in this work a new algorithm which optimizes the measurement procedure and, thus, saves energy towards greener wearables. The main attribute of AHRA is its capability to adapt the sampling frequency to the state of each worker to detect unhealthy conditions which could lead to accidents.

3.3.1. AHRA: Adaptive Heart Rate Algorithm. This section introduces our adaptive heart rate measurement algorithm, AHRA. Since several existing algorithms achieve highprecision measurements at the expense of low energy efficiency, we developed a proprietary algorithm which is able to adapt sampling frequency by performing flexible sleeping periods which does not affect the quality of measurements.

Initially, the algorithm has a one-minute calibration stage for each subject that requires him or her to be quiet and seated to establish a baseline beat-per-minute (bpm) data. The calibration is only performed once per each worker storing the reference value in EEPROM for daily usage. The algorithm identifies voltage peaks and reaches an average peak-to-peak value of time during a 10-second time measurement. Figure 3 shows a flow diagram of AHRA where low-level routines such as calibration or peak determination are omitted.

According to Figure 3, the reference heart rate value obtained is subsequently used for several analyses and, unless the device is hard reset, additional calibrations will not be necessary. The default interval between measurements is initially set to 30 seconds. If the newest value differs from the reference one, AHRA will question whether it has increased or decreased. In case of decrease, the previous interval is broadened by means of a linear equation (factor B in (3)). Since increases in heart rate must be carefully analysed, in those cases a proportional reduction of time between measurements is established according to a polynomial equation reached by experimentation (factor A in (2)). Factor A was determined considering fixed sampling intervals for specific bpm increases; for instance, a 49-second interval was defined for bpm values equal to the base value calibrated while a 2 -second interval was defined for a $10 \mathrm{bpm}$ difference. Considering a set of fixed intervals, the experimental equations (2) and (3) were reached. Furthermore, warning and alert states are defined in AHRA (corresponding to $7 \%$ and $14 \%$ increases in the reference $\mathrm{bpm})$. 


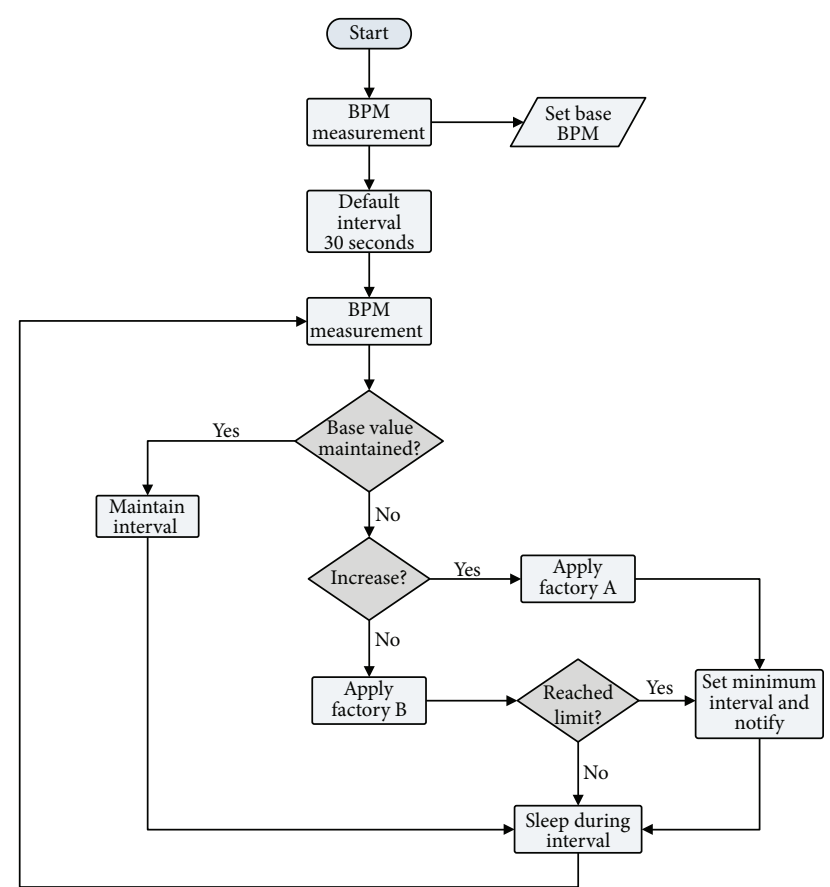

FIGURE 3: Adaptive heart rate algorithm (AHRA).

$$
\begin{aligned}
F_{\mathrm{A}}= & -0.140 \cdot\left(\mathrm{bpm}-\mathrm{bpm}_{\text {base }}\right)^{2} \\
& -3.315 \cdot\left(\mathrm{bpm}-\mathrm{bpm}_{\text {base }}\right)+49, \\
F_{\mathrm{B}}= & \left(\mathrm{bpm}_{\text {base }}-\mathrm{bpm}\right)+50 .
\end{aligned}
$$

\section{Experimental Evaluation and Discussion}

This section addresses a set of experiments carried out using OperaBLE and evaluates the energetic contribution of each algorithm. For carrying out the experiments in OperaBLE, a computing board was used as an external processing device to perform the nonwearable role in a LoMoCA algorithm. The load current was measured to evaluate consumption in different conditions using a shunt resistor and an operational amplifier with the aim of reaching a device lifespan comparison.

In this section, several experiments have been defined to evaluate each algorithm individually and reach their major weaknesses. Section 4.1 is focused on LoMoCA, where five different movements were defined to find singularities depending on the number of planes and rotational axes used during characterisation. Section 4.2 explores the performance of AHRA, and for both algorithms, a brief experiment based on power consumption evaluates the suitability of our proposal.

4.1. LoMoCA Experiments. This section defines five baseline movements related to industry-based tasks, to check as many variants as possible and evaluate the system. Table 2 shows a graph including relevant axes in each case and a simplified trajectory representation. These movements have been classified into two groups: the first one includes the so-called hammer, valve opening and assembly (movements thought to represent common industrial tasks for testing
TABLE 2: Movement patterns for experimentation.

Movement

the algorithm with a progressive difficulty increase) and the second includes data request and notification (which provide support to help operators in their daily work). All movements were recorded as short movements to have a low number of samples and, thus, hinder the characterisation process. A detailed explanation is provided below:

(i) Hammer: uses mainly two axes and only one rotation axis, the 1-2-3 sequence is repeated three times.

(ii) Valve opening: this movement was performed using the OperaBLE XY plane. It deals with an additional difficulty because of the centrifugal acceleration that produces a variation of $\mathrm{Z}$ axis hindering the recognition procedure. The sequence $1-2-3$ is executed four times.

(iii) Assembly: consists of a connection task where 6 axes are relevant (most complex movement), which is useful to check LoMoCA accuracy to distinguish among similar movements. The correct sequence is $1-2-3$.

(iv) Data request: is a special movement recorded to allow operators to request data from the machines using a straightforward method, three taps on the machine surface. It avoids the use of screens, buttons, or complex devices. The sequence $1-2-3$ is repeated three times. 

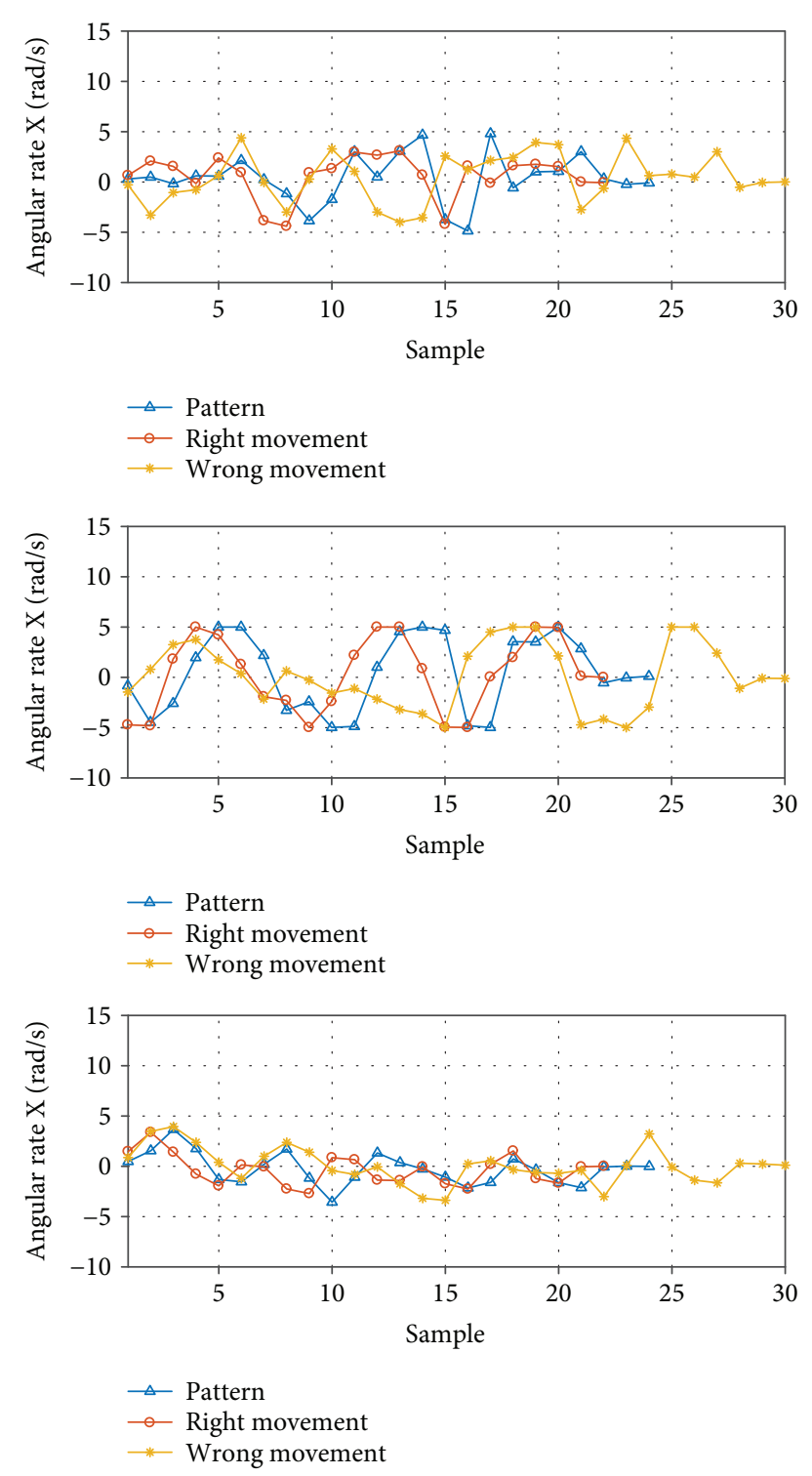

Figure 4: LoMoCA evaluation executing a right and a wrong movement compared to the pattern (gyroscope).

(v) Notification: enables operators to send an emergency message to notify their superior about any issue with no need to abandon the task at hand. It consists of moving the hand in vertical position (1-2 sequence) and shaking it two times (2-3-2 sequence repeated twice).

Assembly movement represents a typical production chain task, where different actions must be realized in the right order. The right sequence of actions is represented in Table 2, and to provide a perspective of data to be analysed by LoMoCA, we represented the angular rate samples of three movements overlapped (see Figure 4): assembly pattern, assembly movement, and wrong assembly movement. All movements have a duration of 2-3 seconds to highlight the low-frequency characterisation. Assembly pattern represents the pattern stored in memory to be used in the algorithm; a

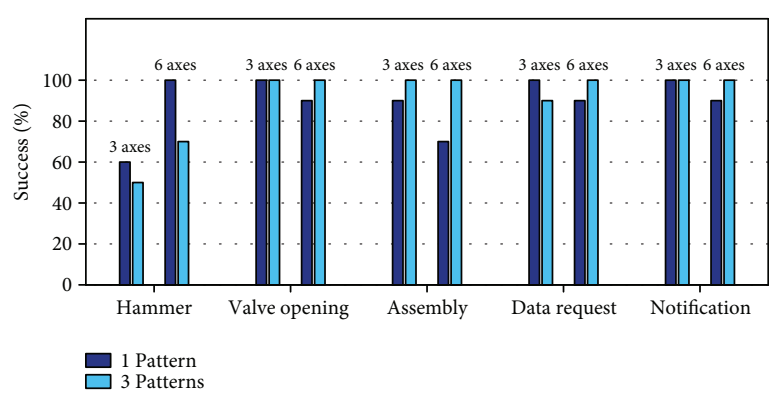

Figure 5: Number of pattern influence in LoMoCA.

right assembly movement follows the right sequence of actions, and wrong assembly refers to the execution of actions in a wrong sequence.

4.1.1. Number of Pattern Influence. LoMoCA introduces as many patterns as required to improve movement characterisation. Since there are many possible manners to execute a movement, the longer the movement is the lower similarity that is appreciated. The baseline idea consists of adding several patterns of the same movement.

The first experiment addresses the influence of the number of patterns stored in characterisation accuracy. Figure 5 shows a statistic with the success rate (using 3 or 6 DOF for each case). The experiment was based on 10 random sequences of the 5 movements recorded to obtain sufficient samples. The results revealed that using only accelerometers lead to wrong hammer characterisations which were identified as data request (see Figure 5). In that case, the use of 3 DOF (only acceleration) is not enough to distinguish between similar movements as hammer and data request. Thus, we concluded that a small number of axes combined with few sample movements increases similarity and consequently, makes LoMoCA select the shortest movement. Thus, the problem of using only acceleration data undermined accuracy increasing false negatives.

This issue was solved by adding the gyroscope data source (6 DOF using 3 linear and 3 rotational axes) to provide extra information, enabling LoMoCA to eradicate false negative characterisations. However, the number of patterns conditioned the success rate depending on the length of each movement (i.e., short movements like hammer do not require a large number of patterns to reach the $100 \%$ according to Figure 5). Therefore, the achievement of a high success rate to satisfy industry requirements is only possible by the use of gyroscope data in this case, being present the mentioned low-frequency restriction.

The addition of extra DOF by including gyroscopes is helpful for improving characterisation of simple movements, at the expense of a higher energy consumption. As stated in previous sections, the key priority of the system is reliability in order to enable the use of OperaBLE in Industry 4.0 scenarios. Thus, the extra power demand required by the addition of gyroscopes is justified to achieve a proper characterisation in the case of short movements. However, depending on the final application of OperaBLE, the use of 3 or $6 \mathrm{DOF}$ is an aspect to be carefully 
TABle 3: Assembly successful recognition using 3 or 6 axes.

\begin{tabular}{lcccc}
\hline \multirow{2}{*}{ Sequence } & \multicolumn{2}{c}{3 axes } & \multicolumn{2}{c}{6 axes } \\
& Output & Success & Output & Success \\
\hline $1-2-3$ & $\checkmark$ & $\checkmark$ & $\checkmark$ & $\checkmark$ \\
$(\checkmark)$ & $\checkmark$ & $\checkmark$ & $\checkmark$ & $\checkmark$ \\
$3-2-1$ & $\checkmark$ & $x$ & $x$ & $\checkmark$ \\
$(\boldsymbol{x})$ & $\boldsymbol{x}$ & $\checkmark$ & $x$ & $\checkmark$ \\
$2-3-1$ & $\boldsymbol{x}$ & $\checkmark$ & $x$ & $\checkmark$ \\
$(\boldsymbol{x})$ & $\boldsymbol{x}$ & $\checkmark$ & $x$ & $\checkmark$ \\
$3-1-2$ & $\boldsymbol{x}$ & $\checkmark$ & $x$ & $\checkmark$ \\
$(\boldsymbol{x})$ & $\boldsymbol{x}$ & $\checkmark$ & $\boldsymbol{x}$ & $\checkmark$ \\
$2-1-3$ & $\checkmark$ & $\boldsymbol{x}$ & $\checkmark$ & $\boldsymbol{x}$ \\
$(\boldsymbol{x})$ & $\checkmark$ & $\boldsymbol{x}$ & $\boldsymbol{x}$ & $\checkmark$ \\
$1-3-2$ & $\checkmark$ & $\boldsymbol{x}$ & $\boldsymbol{x}$ & $\checkmark$ \\
$(\boldsymbol{x})$ & $\boldsymbol{x}$ & $\checkmark$ & $\boldsymbol{x}$ & $\checkmark$ \\
\hline & & $66.7 \%$ & & $91.7 \%$ \\
\hline
\end{tabular}

considered, according to the results of this experiment and the energetic restrictions.

4.1.2. Assembly Stress Test. The methodology of this experiment consisted of executing twice assembly in all possible wrong sequence combinations (3-2-1, 2-3-1, etc.), considering both 3 and 6 DOF to compare the influence of using more DOF in our algorithm. Considering the 1-2-3 sequence as correct (see Table 2), Table 3 provides the results of our assembly stress test where remarkable conclusions have been reached.

Using $3 \mathrm{DOF}$, the right movement (sequence 1-2-3) is correctly characterised in both repetitions. Nevertheless, we detected a false-positive issue since assembly was recognised in 4 occasions erroneously. Consequently, the addition of 3 extra DOF improved significantly the results, increasing the success rate from $66.7 \%$ to $91.7 \%$. This fact enables the use of LoMoCA for more complex actions where the sequence of the movement is critical for the proper functioning of the process. This supports the idea that gyroscopes are essential, depending on the final application, to improve the performance of OperaBLE. Thus, only in situations where the addition of these 3 extra DOF is required to ensure reliability, the consumption of the device will be increased by the addition of a gyroscope data source.

4.1.3. Power Awareness: LoMoCA. This section offers an analysis of OperaBLE regarding power consumption during LoMoCA (varying number of DOF for different movements). The sampling frequency for consumption measurements was $10 \mathrm{~Hz}$, and the source connected to OperaBLE was a $105 \mathrm{mAh}$ battery. The experiment was performed during the execution of our reference movements (see Table 2); the numerical values were stored, and the consumption curve was obtained.

The average current measured during the characterisation stage for hammer, valve opening, and assembly movements was practically constant, since the duration of each movement had a greater impact on the consumption than

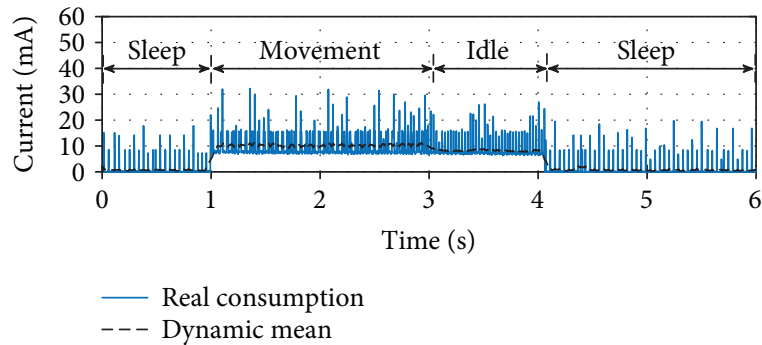

Figure 6: Average consumption per movement (hammer) in a 6-second interval using LoMoCA.

the number of iterations performed (determined by the kind of movement executed). Thus, our aim remained on the determination of the current consumed during the processing stage of a movement as well as the time interval required for characterisation.

The consumption curve of OperaBLE during hammer movement is shown in Figure 6, where the stages identified are sleep, movement, and idle. In this representation, OperaBLE characterised correctly the movement by only using 3 DOF (acceleration data). Additionally, it was maintained in a sleep state before and after characterisations (movement stage) to improve the identification of the different consumption stages, leaving a 1-second idle stage to ensure that the movement data gathering had finished. In the case of valve opening and assembly movements, the time spent during the movement reached approximately 3 seconds. Thus, OperaBLE remained 2 seconds in a sleep mode in these cases, where the sleeping time coincided with the movement time for a duty cycle.

The time spent during the movement characterisation is a relevant factor to consider. For the 6-second measurement interval defined in this experiment, OperaBLE consumes, at most (assembly movement), a capacity of $0.011 \mathrm{mAh}$. This means that our prototype will operate under such work flow for at least 15.70 hours while using 6 DOF (worst case). A lifespan of 10.27 hours is obtained dividing the $105 \mathrm{mAh}$ capacity of our battery by the average consumption of $10.22 \mathrm{~mA}$ (movements being constantly characterised), which is the overall worst-case scenario. Even though this estimation is based on real measurements, the lifespan reached is ideal. For a $30 \%$ lifespan attenuation, the device would still operate for a complete working day with no energy constraints.

Apart from the time spent by each movement characterisation, this work proposes a set of priorities and decision-making procedure oriented to the number of DOF to be considered to save as much energy as possible. As stated in previous sections, the key priority for OperaBLE is reliability in order to fulfil the requirements of Industry 4.0. However, sustainability is as well an essential aspect for the algorithms, for which two main sources of energy consumption exist: the operating frequency of the microcontroller of the device (on which this work focuses) and the sensor modules attached to the device. Since communication management does not conflict with the previous, we highlight in the following lines the two 


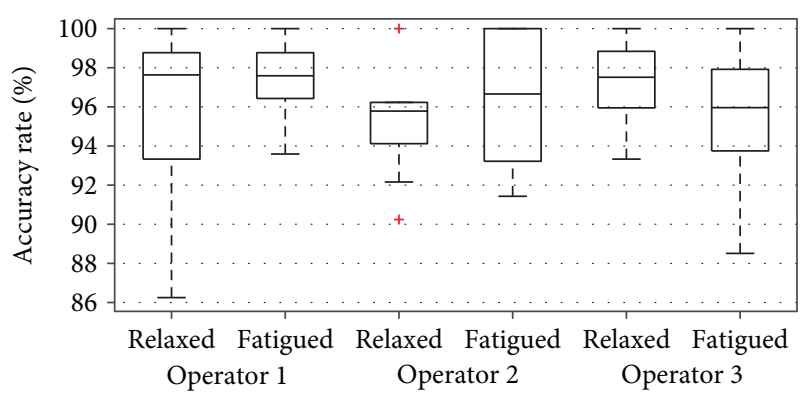

FIGURE 7: Statistical accuracy rate (\%).

alternatives explored regarding the consumption sources for OperaBLE:

(1) The use of a high-frequency microcontroller, which would not require in many situations the addition of gyroscope data at the expense of a higher global consumption of the device. According to the technical datasheet of the IMU used, the consumption of the gyroscope is noticeably higher than the consumption of the accelerometer, which is the reason why avoiding the use of these extra DOF could contribute to greater energy savings in the sensor board. This alternative was discarded to focus on the microcontroller as the energy saving source for OperaBLE, which is the objective of this work and is expected to provide greater energy savings.

(2) The implementation of the system with a lowfrequency limitation applied to the microcontroller, at the expense of adding in certain situations 3 extra DOF provided by the gyroscope to satisfy the reliability requirement. Although the sensor consumption would be increased, the global consumption of the microcontroller board can be considerably reduced, which affects the overall efficiency of the device. Focusing on this idea, this work proposes the reduction of power consumption based on the implementation of LoMoCA with this low-frequency restriction.

As a result, since the experiments carried out highlight a necessity of adding gyroscope data in short movements such as hammer (to satisfy the reliability requirement), the algorithm was developed to be easily adaptable to the final application. Thus, 3 DOF can be used in applications where LoMoCA does not require gyroscope data to successfully characterise movements (i.e., valve opening, data request, or notification) and 6 DOF will be used in situations where the reliability of characterisation may be undermined by this low-frequency requirement. On the whole, the sustainability of the proposal is based on the low-frequency operation of lightweight devices highly compatible with LoMoCA and enhanced by the addition or removal of angular velocity measurements depending on the necessities.

4.2. AHRA Experiments. This section focuses on AHRA, our adaptive heart rate algorithm, to test the accuracy of

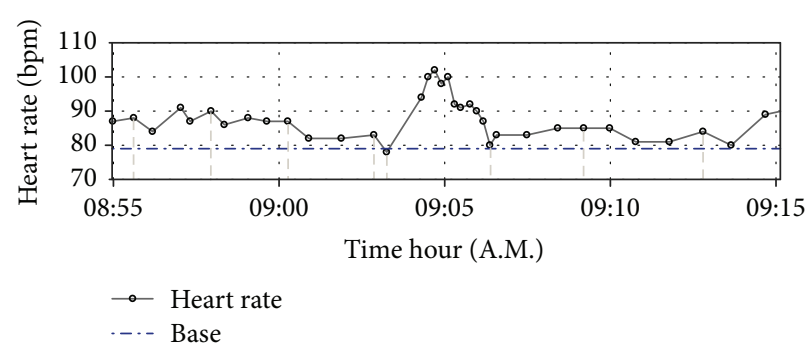

(a)

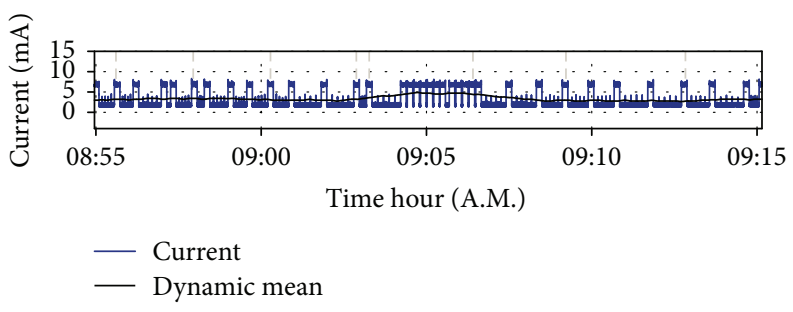

(b)

FIgURE 8: Heart rate test and simultaneous consumption.

measurements provided by OperaBLE and the adaptability of the prototype to different workers and conditions.

4.2.1. Adaptive Heart Rate Algorithm Measurements. Three different workers were selected to carry out the first experiment, which consisted of extracting a bpm measurement while performing different tasks. The measurements where stored and compared to the ones calculated by characterising the average peak-to-peak time with a digital oscilloscope.

During the experiment, the calibration routine defined in AHRA was used with three different test workers, 10 times for each subject. Eventually, they were asked to fulfil a form indicating whether they had felt fatigued or relaxed. The percentage of statistical accuracy for each group of 10 measurements has been represented in Figure 7, where fatigued and relaxed categories are highlighted for each operator.

The success accuracy rate (see Figure 7) shows the proximity between the measured and the real bpm value. The results of the experiment have been classified into 6 groups according to the state of each operator. The highest dispersion is noticed in operator 1 (relaxed), where the quartiles range from $86 \%$ to $100 \%$ of accuracy rate. The statistical representation of the relaxed state of operator 2 clearly shows that our algorithm has a good degree of repeatability (see quartiles 2 and 3 in Figure 7).

AHRA is suitable for measuring the heart rate in different conditions and workers. The measured value coincided with the expected in repeated occasions, and the average accuracy rate of success reached during the experiment was $96.16 \%$. Since the main aim of OperaBLE is to identify risky situations, the algorithm is accurate and appropriate for detecting harmful changes to notify nearby supervisors.

4.2.2. Power Awareness: AHRA. This section describes an experiment based on a power-aware approach to quantify the energy savings of adapting sampling frequency to the 
state of each worker. For this, a specific operator was subjected to differentiated emotional states triggered by external agents. The resulting data is shown in Figure 8(a), where a 20 -minute time interval has been represented to focus on the most representative attribute of AHRA (adaptability). The part of Figure 8(b) includes the consumption obtained while measuring heart rate in OperaBLE to remark sustainability. It can be noticed how OperaBLE turns into sleep mode after each measurement, adjusting the sleeping interval (according to (1) and (2)).

Before starting to measure heart rate (08:54 A.M.), the operator was asked to calibrate OperaBLE to define a reference bpm value. The measurements shown were obtained from 08:55 A.M. to 09:15 A.M., time interval in which the test worker was performing a predefined task, and ten minutes after starting to store measurements (around 09:05 A.M.), an external agent provoked an unexpected situation. Consequently, the average bpm increased instantaneously and we could verify how the algorithm applied factor A (1) to reduce drastically the sleeping interval. Eventually, the subject returned to normal bpm values and OperaBLE augmented the sampling interval to save as much energy as possible (09:10 A.M.).

According to this experiment, we conclude that OperaBLE has an instantaneous current of $3 \mathrm{~mA}$ during relaxed situations while the consumption could be increased up to $7 \mathrm{~mA}$ when identifying fatigue or stress at work. Thus, OperaBLE achieves the sustainability challenge and ensures a minimum 15-hour lifespan (up to 35 hours in nonharmful situations).

\section{Conclusions and Future Work}

This research is motivated by the most challenging goals of the emerging industrial paradigm, the so-called Industry 4.0, where IoT technologies will play an essential role towards smart factories. In order to enhance working conditions and improve efficiency at work, this paper introduces our factory-wearable prototype, OperaBLE. This proposal is due to track operators in smart factories, avoiding accidents at workplaces and identifying harmful working conditions.

The major achievement of this work is the introduction of two power-aware algorithms, AHRA and LoMoCA, which have been specifically developed to operate in lowcost and low-power devices such as OperaBLE. These algorithms are based on flexible approaches in order to face the Industry 4.0 sustainability challenge. A set of experiments were carried out to evaluate these algorithms separately and verify the reliability and lifespan of the OperaBLE device during a working day.

Regarding LoMoCA, we reached the conclusion that the combination of accelerometers and gyroscopes (considering one pattern for simple movements and three for complex actions) conformed the best configuration achieving a $96 \%$ of success in an average situation, at the expense of greater energy consumption caused by gyroscopes. However, the use of this extra sensor is only justified in the case of specific situations such as short or sequence-sensitive movements, which could create confusion to the algorithm. Therefore, depending on the final application and the kind of movements to be characterised, a higher energy demand will be required not to compromise reliability for industrial applications, which is a key priority.

OperaBLE is flexible enough to characterise accurately any movement using low frequency, which enables its use in lightweight devices contributing towards a better use of the resources and an enhanced energy efficiency. The acceleration-based system developed permits data request operations by tapping on the surface of machines, avoiding the use of screens, or complex interfaces. It enables operators to send supervisors movement-based notifications immediately in case of emergency, which is essential to prevent accidents at work.

Regarding well-being in smart factory spaces, three workers were selected for evaluating AHRA in different states. Measurements presented an average accuracy rate of success $96.16 \%$ using OperaBLE with respect to measurements provided by a digital oscilloscope, which enabled OperaBLE to identify harmful conditions for workers such as stress or fatigue. Furthermore, thanks to the adaptable behaviour of AHRA, the average current consumed during heart rate measurements was minimal ( $3 \mathrm{~mA}$ approximately) while the low-frequency operation of LoMoCA allowed OperaBLE to reach a 10.27-hour lifespan in a worst-case scenario of constant movement characterisation and using a 105 mAh battery capacity.

Future works include a redesign of the prototype in order to reduce its size and enhance comfortability for operators. More algorithms are currently being developed to empower modularity in OperaBLE and track the surrounding environments of operators ensuring safety at work. Since BLE technology enriches scalability and offers possibilities for largerscale applications, a BLE mesh network is being deployed at the moment for evaluating a complete BLE-based CPS. The results achieved are promising, deserving further research to evaluate robustness and increases productivity in real Industry 4.0 environments.

\section{Data Availability}

The data that support the findings of this study are available from the corresponding author, Diego Hortelano, upon reasonable request.

\section{Conflicts of Interest}

The authors declare that there is no conflict of interest regarding the publication of this paper.

\section{Acknowledgments}

This work was supported by the Spanish Ministerio de Eduación, Cultura y Deportes under Grants TIN201566972-C5-2-R (MINECO/FEDER) and TIN2015-65845C3-2-R and also by the I+D+i Universidad de Castilla-La Mancha plan with the European Social Fund. 


\section{References}

[1] Y. Lu, "Industry 4.0: a survey on technologies, applications and open research issues," Journal of Industrial Information Integration, vol. 6, pp. 1-10, 2017.

[2] E. Borgia, "The Internet of Things vision: key features, applications and open issues," Computer Communications, vol. 54, pp. 1-31, 2014.

[3] R. Rajkumar, I. Lee, L. Sha, and J. Stankovic, "Cyber-physical systems: the next computing revolution," in DAC '10 Proceedings of the 47th Design Automation Conference, pp. 731-736, New York, NY, USA, June 2010.

[4] Z. A. Shhedi, A. Moldoveanu, F. Moldoveanu, and C. Taslitchi, "Real-time hand hygiene monitoring system for HAI prevention," in 2015 E-Health and Bioengineering Conference (EHB), pp. 1-4, Iasi, Romania, November 2015.

[5] F. Cai, C. Yi, S. Liu et al., "Ultrasensitive, passive and wearable sensors for monitoring human muscle motion and physiological signals," Biosensors and Bioelectronics, vol. 77, no. 15, pp. 907-913, 2016.

[6] K. Niazmand, K. Tonn, Y. Zhao et al., "Freezing of gait detection in Parkinson's disease using accelerometer based smart clothes," in 2011 IEEE Biomedical Circuits and Systems Conference (BioCAS), pp. 201-204, San Diego, CA, USA, November 2011.

[7] R. Brugarolas, J. M. Valero-Sarmiento, and A. Brna, "Wearable $\mathrm{SpO}_{2}$ and sleep posture monitoring system for obstructive sleep apnea patients," in 2015 IEEE Virtual Conference on Applications of Commercial Sensors (VCACS), pp. 1-6, Raleigh, NC, USA, March 2015.

[8] R. Abbasi-kesbi and A. Nikfarjam, "A mini wearable wireless sensor for rehabilitation applications," in 2015 3rd RSI International Conference on Robotics and Mechatronics (ICROM), pp. 618-622, Tehran, Iran, October 2015.

[9] M. Nguyen, L. Fan, and C. Shahabi, "Activity recognition using wrist-worn sensors for human performance evaluation," in 2015 IEEE International Conference on Data Mining Workshop (ICDMW), pp. 164-169, Atlantic City, NJ, USA, November 2015.

[10] R. San-Segundo, J. Lorenzo-Trueba, B. Martinez-Gonzalez, and J. M. Pardo, "Segmenting human activities based on HMMs using smartphone inertial sensors," Pervasive and Mobile Computing, vol. 30, pp. 84-96, 2016.

[11] J. S. Park, S. Robinovitch, and W. S. Kim, "A wireless wristband accelerometer for monitoring of rubber band exercises," IEEE Sensors Journal, vol. 16, no. 5, pp. 1143-1150, 2016.

[12] K. J. Sheehan, B. R. Greene, C. Cunningham, L. Crosby, and R. A. Kenny, "Early identification of declining balance in higher functioning older adults, an inertial sensor based method," Gait \& Posture, vol. 39, no. 4, pp. 1034-1039, 2014.

[13] R. C. Van Lummel, E. Ainsworth, U. Lindemann et al., “Automated approach for quantifying the repeated sit-to-stand using one body fixed sensor in young and older adults," Gait \& Posture, vol. 38, no. 1, pp. 153-156, 2013.

[14] S. Urmat and M. E. Yalçın, "Design and implementation of an ARM based embedded system for pedestrian dead reckoning," in 2015 9th International Conference on Electrical and Electronics Engineering (ELECO), pp. 885-889, Bursa, Turkey, November 2015.

[15] W. Lee, E. Seto, K.-Y. Lin, and G. C. Migliaccio, “An evaluation of wearable sensors and their placements for analyzing construction worker's trunk posture in laboratory conditions," Applied Ergonomics, vol. 65, pp. 424-436, 2017.

[16] W. Lee, K.-Y. Lin, E. Seto, and G. C. Migliaccio, "Wearable sensors for monitoring on-duty and off-duty worker physiological status and activities in construction," Automation in Construction, vol. 83, pp. 341-353, 2017.

[17] J. Chen, J. Qiu, and C. Ahn, “Construction worker's awkward posture recognition through supervised motion tensor decomposition," Automation in Construction, vol. 77, pp. 67-81, 2017.

[18] R. Akhavian and A. H. Behzadan, "Smartphone-based construction workers' activity recognition and classification," Automation in Construction, vol. 71, pp. 198-209, 2016.

[19] M. Oliver, G. M. Schofield, H. M. Badland, and J. Shepherd, "Utility of accelerometer thresholds for classifying sitting in office workers," Preventive Medicine, vol. 51, no. 5, pp. 357360, 2010.

[20] Punch Through, "LightBlue family2018, http://www. punchthrough.com/bean.

[21] K. Mikhaylov and J. Tervonen, "Optimization of microcontroller hardware parameters for wireless sensor network node power consumption and lifetime improvement," in International Congress on Ultra Modern Telecommunications and Control Systems, pp. 1150-1156, Moscow, Russia, October 2010.

[22] H. Sakoe and S. Chiba, "Dynamic programming algorithm optimization for spoken word recognition," IEEE Transactions on Acoustics, Speech, and Signal Processing, vol. 26, no. 1, pp. 43-49, 1978.

[23] C. F. S. Souza, C. E. P. Pantoja, and F. C. M. Souza, Verificação de Assinaturas Offline Utilizando Dynamic Time Warping, Universidade de Brasília, 2015.

[24] E. Langholz, "Dynamic time warping," 2014, http://github. com/langholz/dtw.

[25] D. Hortelano, T. Olivares, M. C. Ruiz, C. Garrido-Hidalgo, and V. López, "From sensor networks to Internet of Things. Bluetooth Low Energy, a standard for this evolution," Sensors, vol. 17, no. 2, pp. 1-31, 2017. 


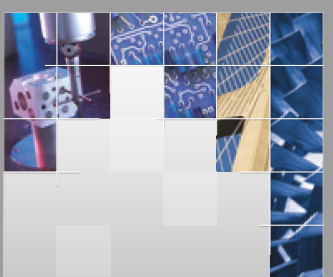

\section{Enfincering}
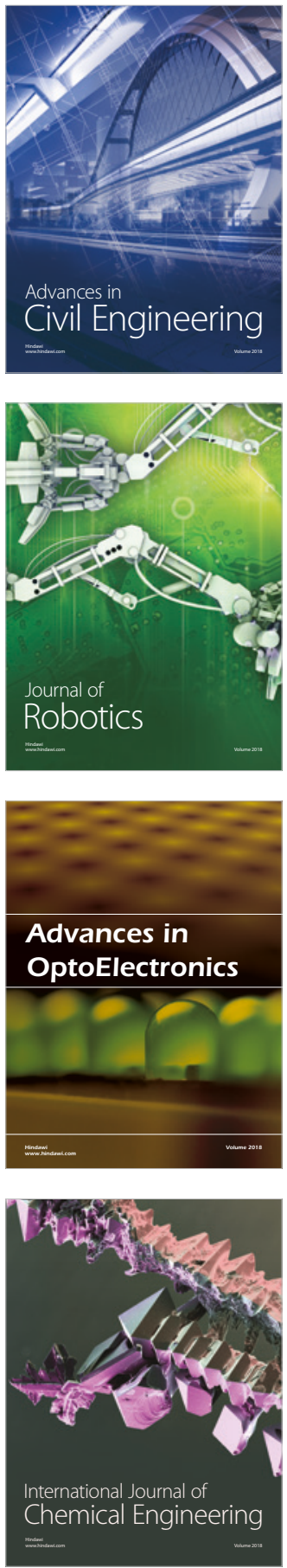

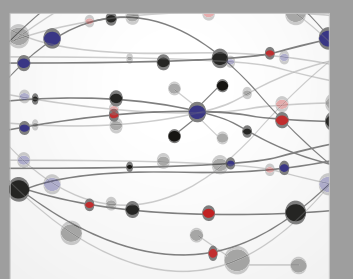

\section{Rotating \\ Machinery}

The Scientific World Journal

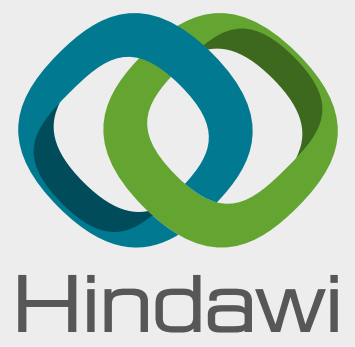

Submit your manuscripts at

www.hindawi.com
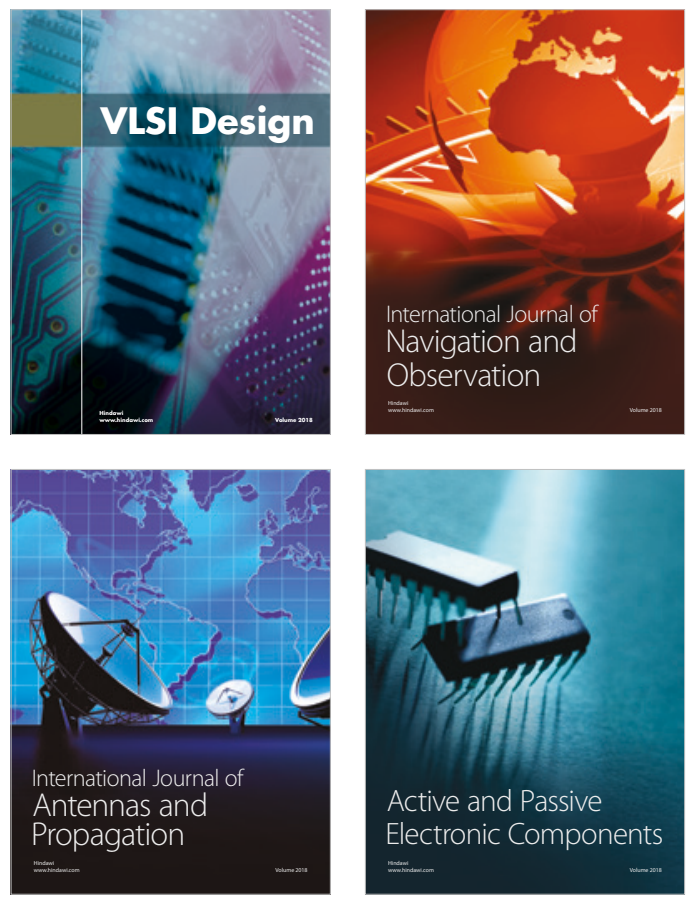
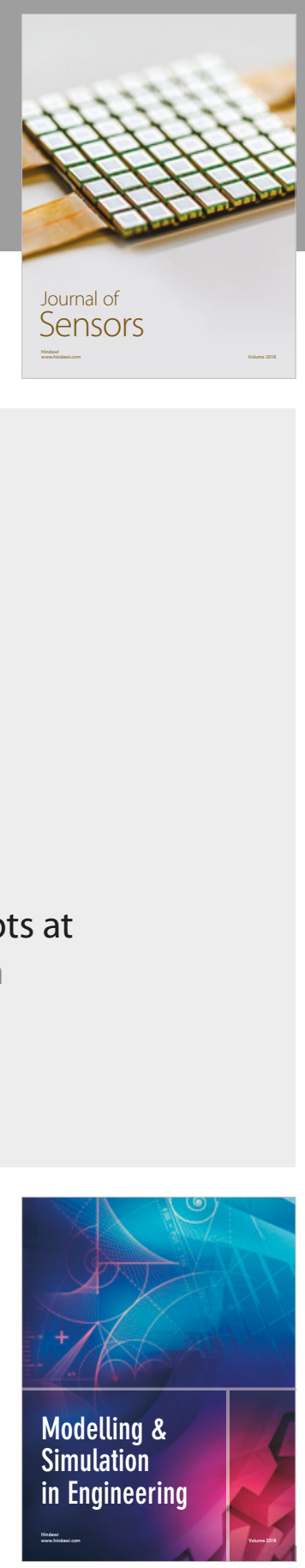

\section{Advances \\ Multimedia}
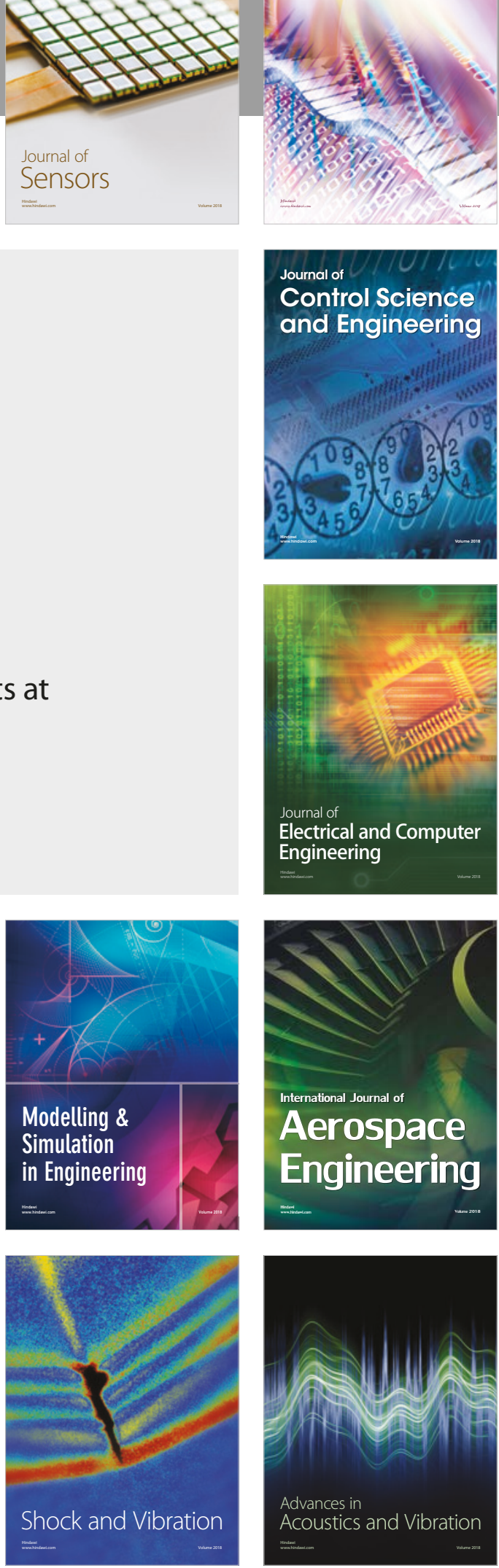